\title{
Dietary patterns and glycaemic control among Qatari adults with type 2 diabetes
}

\author{
Marwa Alaradi, Asma Ouagueni, Rim Khatib, Grace Attieh, Hiba Bawadi and \\ Zumin Shi* \\ Human Nutrition Department, College of Health Sciences, QU Health, Qatar University, P.O. Box 2713, Doha, Qatar
}

Submitted 28 March 2020: Final revision received 20 August 2020: Accepted 10 September 2020

\begin{abstract}
Objective: To assess the association between dietary patterns and glycaemic control among Qatari adults with type 2 diabetes (T2DM).

Design: Cross-sectional analysis using data from the Qatar Biobank Study. Poor glycaemic control was defined as HbA1c $\geq 7 \cdot 0 \%$. Dietary patterns were constructed using factor analysis based on habitual food intake assessed by a FFQ. Medication use was based on self-report. Multivariable logistic regression was used to assess the association.

Setting: Qatar.

Participants: Adults aged $\geq 18$ years ( $n$ 1000) with known diabetes.

Result: The mean age of the participants was 52.3 (SD 11.5) years. Overall, the prevalence of poor glycaemic control was $57.6 \%$, and $27.7 \%$ of the participants were insulin users. Three dietary patterns were identified. The modern dietary pattern (high intake of fast food, croissants, white bread and cheese) was inversely associated with poor glycaemic control. The SD increments of the modern pattern had OR for poor glycaemic control of 0.86 (95\% CI $0.68,1.08)$ in men and 0.76 (95\% CI 0.61, 0.95) in women. There was a significant interaction between the modern pattern and diabetes medication in men but not in women. In men without diabetes medication, the modern pattern was positively associated with poor glycaemic control with an OR of $2 \cdot 35$ (95\% CI 1.13, 4.87).

Conclusions: Male diabetes patients took medication to control diabetes but ate more unhealthy food. In men who were not taking diabetes medication, modern dietary pattern was associated with poor glycaemic control. Promoting healthy eating should be encouraged especially among those under diabetes medication.
\end{abstract}

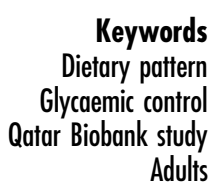

The prevalence of type 2 diabetes (T2DM) has been increasing globally with approximately 425 million adults affected in 2017, and this number will reach 629 million by $2045^{(1)}$. The Middle East and North Africa region has the highest prevalence of diabetes in the world ${ }^{(1)}$. The latest data from the Qatar Biobank (QBB) study showed that the prevalence of diabetes mellitus in adults was $17 \cdot 4 \%{ }^{(2)}$. The high prevalence of diabetes brings a substantial economic and health burden to society ${ }^{(3)}$, including CVD and premature death ${ }^{(4)}$.

Poor control of diabetes is common and increases the risk of complications such as neuropathy disease, nephropathy, retinopathy and $\mathrm{CVD}^{(5)}$. More than $60 \%$ of patients in the UAE, Bahrain, Saudi Arabia and Kuwait exhibited poor glycaemic control ${ }^{(6-8)}$. In Qatar, about $50 \%$ of people with diabetes had poor glycaemic control as defined as HbA1c above $7 \mathrm{mmol} / \mathrm{l}^{(9)}$. Although genetics can affect the risk of diabetes and glycaemic control, lifestyle factors such as diet, physical activity, smoking and alcohol consumption are also associated with glycaemic control ${ }^{(10-14)}$. Dietary factors, such as high intake of fruits, vegetables, and fish, the Mediterranean diet, DASH diet can help glycaemic control, while high intake of saturated fats, salt and energy-dense Western diet is associated with poor glycaemic control ${ }^{(15-17)}$. One of the mechanisms by which poor diet affects glycaemic control is through obesity. Weight loss is a key determinant for glycaemic control among those with diabetes ${ }^{(18)}$. A network meta-analysis of fifty-six clinical trials found that compared with other eight dietary approaches (low-fat, Vegetarian, Mediterranean, high-protein, moderate-carbohydrate, low-carbohydrate, control, low glyacemic index (GI) and 
glycaemic load (GL), Palaeolithic), the Mediterranean diet is the most effective and efficacious dietary approach to improve glycaemic control in type 2 diabetes ${ }^{(19)}$. However, none of the fifty-six studies was conducted in the Arabic countries. As dietary habit is culture-specific, identification of dietary patterns which may affect glycaemic control is a key for diabetes management.

Similar to other Arabic countries, the diet in Qatar is characterised by high intake of energy and sugar. Based on a national survey, the mean intake of sugar was $153 \mathrm{~g} / \mathrm{d}$ among Qatari residents ${ }^{(20)}$. Moreover, the intake of meat, refined carbohydrates and $\mathrm{Na}$ was high, while the intake of $\mathrm{Ca}$ and fibre-rich sources like fruits and vegetables was low ${ }^{(20)}$. Purchased food in Qatari households contains double the amount of protein and energy requirements, and the high intake of sugars and fat is due to the subsidy programme in the country ${ }^{(20)}$.

Unhealthy diet and a sedentary lifestyle contribute to the high prevalence of diabetes and obesity in Qatar ${ }^{(21)}$. More than $70 \%$ of adults in Qatar were found to be overweight or obese $^{(22)}$. Posterior (data-driven approach as compared with knowledge-based method) dietary patterns using factor analysis, cluster analysis or reduced rank regression have been increasingly used in nutritional epidemiology as they may assess the overall dietary quality and reflect the interactions between foods and nutrients in the diet ${ }^{(23,24)}$. Little is known about the relationship between dietary patterns and glycaemic control in Qatar. So far, only one study has assessed the association between dietary patterns and glycaemic control based on the QBB study ${ }^{(9)}$. Low intake of Mg-related dietary pattern derived by reduced rank regression has been shown to be associated with poor glycaemic control $^{(9)}$. No study has examined the interaction between dietary patterns and medication use in relation to glycaemic control. To address these issues, the aim of this study was to assess the association between dietary patterns using factor analysis, and glycaemic control among Qatari adults with type 2 diabetes.

\section{Methods}

\section{Study design and sample}

The QBB study is an ongoing population-based cohort study that started in 2012. It recruits those who have lived in Qatar for 15 years or more and are over the age of 18 years. The recruitment of participants was through the Internet, family and friends, or via social media. The study included a self-administered questionnaire and nurse interview. Data about sociodemographic information, lifestyle factors and dietary habits were collected using the questionnaire; data about family history of disease, health conditions and the use of medication were collected during the nurse interview with the participants. The participants were invited to the QBB facility in the Hamad Medical City to have all the health examinations done. Participants' weight and height were measured using a Seca stadiometer by the research nurses. Furthermore, $60 \mathrm{ml}$ of blood samples was taken and measured for a total of sixty-six biomarkers at the QBB facility ${ }^{(25)}$.

In the current study, a random sample of 1000 Qatari participants (500 men and 500 women) was selected from the whole database by the QBB management group. The sample size was determined by the fact that QBB provides 1000 samples to research projects conducted at institutions in Qatar for free. Selected participants included those aged 18 years and above with known diabetes who completed the FFQ and had complete exam data.

\section{Outcome variables: poor glycaemic control as defined by $\mathrm{HbA1c}$}

Self-reported diabetes and diabetes-related information were determined based on the following questions: 'Has a doctor ever told you that you had or have diabetes?'; 'How old were you when your diabetes was first diagnosed?'; 'Are you being treated for your diabetes? (1) No; (2) Don't know; (3) Prefer not to answer; (4) Diet; (5) Increased physical activity; (6) Tablets; (7) Insulin.' and 'Did you start insulin within one year of your diagnosis of diabetes?'. Blood samples were measured for HbA1c and glucose levels. Poor glycaemic control was defined as $\mathrm{HbA} 1 \mathrm{c} \geq 7 \cdot 0 \%$.

\section{Exposure variable: dietary patterns}

Dietary intake was assessed by a self-administered computerised FFQ. The dietary questionnaire was asked about dietary habits as well as modifications and consumption frequency of foods and beverages over the previous year ${ }^{(2)}$. The FFQ included 102 food items and was adapted from the European Prospective Investigation into Cancer and Nutrition (EPIC) study; it has not been validated in Qatar. In the analysis, we grouped the items into thirty-eight food groups based on similarities of nutrient profiles or cooking methods between the food items (see online supplementary material, Supplemental Table 1). Frequency intake (times/week) of the thirty-eight food groups was used as the input variable to construct dietary patterns using factor analysis. The following criteria were used in determining the number of dietary patterns: (1) eigenvalue $>1$; (2) a scree plot (see online supplementary material, Supplemental Fig. 1) and (3) the patterns should be interpretable based on the Qatar food culture. Varimax rotation was used to help the interpretation of the identified patterns. Participants were assigned pattern-specific factor scores. Scores for each pattern were calculated as the sum of the products of the factor loading coefficients and standardised weekly frequency intake of each food group associated with that pattern.

\section{Covariates}

The following variables were considered as covariates: education (low $=$ primary and secondary school; medium = technical or professional school; high $=$ university and 
postgraduate degree), age, gender, smoking and leisure time physical activity level. Physical activity (metabolic equivalent (MET) h/week) was assessed by the International Physical Activity Questionnaire. Participants with a BMI of $25-29.9 \mathrm{~kg} / \mathrm{m}^{2}$ were defined as overweight. Obesity was defined as BMI of $\geq 30 \mathrm{~kg} / \mathrm{m}^{2}$.

\section{Statistical analysis}

To compare the differences between groups, the $\chi^{2}$ test was used for categorical variables and ANOVA for continuous variables. To assess the association between dietary patterns and glycaemic control, multivariable logistic regression was used. Two models were used: model 1 adjusted for age and gender; model 2 further adjusted for smoking, education, physical activity, BMI (continuous) and hypertension. Multiplicative interaction between gender, diabetes medication usage, diabetes duration ( $<10$ years or $\geq 10$ years) and dietary patterns was tested by adding a product term of these variables in a multivariable logistic regression model. A marginsplot command in Stata was used to visualise some of the interactions. Treating diabetes duration as a continuous variable did not change the findings. For simplicity, we categorised diabetes duration as a categorical variable. We did not conduct multiple imputation analysis as only two participants had missing values of food intake in some food items and were treated as no consumption. In total, fourteen men and 116 women had missing information on smoking and treated as nonsmoking. We did not adjust for medication in multivariable model due to the interaction between diabetes medication and dietary pattern. All the data analyses were performed using STATA 15 (Stata Corporation), and statistical significance was considered at $P$-value $<0.05$ (two-sided).

\section{Results}

The mean age of the participants was 52.3 (SD 11.5) years. Overall, the prevalence of poor glycaemic control was $57.6 \%$ (60.8\% in men and $54.4 \%$ in women) (Table 1$)$. The mean diabetes duration was 12.6 (SD 9.4) years among 788 participants who reported duration. Overall, $27.7 \%$ of the participants were insulin users $(25.6 \%$ in men and $29.8 \%$ in women), and $74 \%$ of men and women reported on a diet to prevent/manage chronic conditions. The prevalence of obesity was $44.0 \%$ in men and $70.4 \%$ in women.

Three dietary patterns were obtained (Table 2). Factor 1 ('Modern') was loaded heavily on croissants, white bread and cheese, breakfast cereal, butter, fast food and ice cream. Most of these food items are made from refined grains and sugar. Factor 2 ('Prudent') was characterised by a high intake of salad and raw vegetables, fresh fruit and canned or dried fruit and dates, cooked vegetables, yoghurt, and fish. Factor 3 ('Traditional Food') was mainly contributed by chicken, meat, fish dishes, biryani and fast food, Asian noodle, red meat, white rice and desserts. The three factors explained $14.5 \%$ of the variance in intake.

Supplement Table 2 shows the sample characteristics by levels of dietary pattern intakes. Compared with low intake of the modern dietary pattern, high intake was associated with high education levels and supplement use, but participants were less likely to have hypertension, poor glycaemic control or use diabetes and hypertension medications. Participants with high intake of the prudent dietary pattern were more likely to be highly educated, use supplements and frequent intake of fruits and vegetables. Age was inversely associated with modern dietary pattern intake but positively associated with traditional dietary pattern and prudent pattern intake.

The modern dietary pattern was inversely associated with poor glycaemic control in men but not in women after adjusting for age (Table 3). In the maximally adjusted model, for each unit increase of modern dietary pattern score, the OR for poor glycaemic control was 0.86 (95\% CI $0.68,1.08)$ in men, 0.76 (95\% CI $0.61,0.95)$ in women and 0.80 (95\% CI 0.68, 0.93) in genders combined. No significant associations between the prudent pattern and the traditional pattern with glycaemic control were found.

There was a significant interaction between gender, diabetes medication usage, with the modern dietary pattern in relation to poor glycaemic control ( $P$ for three-way interaction 0.006) (Fig. 1). In men, the modern dietary pattern was positively (OR 2.35 (95\% CI 1.13, 4.87)) associated with poor glycaemic control among those taking diabetes medication but inversely associated with poor glycaemic control among those without diabetes medication. There was also a significant interaction between the traditional dietary pattern and diabetes medication use in relation to poor glycaemic control in men ( $P$ for dietary pattern and diabetes medication two-way interaction in men 0.002); the pattern was positively associated with poor glycaemic control among those with diabetes medication but inversely associated with poor glycaemic control among those without diabetes medication. In women without diabetes medication, the prevalence of poor glycaemic control was low at all levels of dietary patterns intake.

\section{Discussion}

In this cross-sectional study of 1000 people with known diabetes, we constructed dietary patterns using factor analysis and examined the association between the identified dietary patterns and glycaemic control. The modern food pattern characterised by high intake of fast food, sugar and refined grains was inversely associated with poor glycaemic control. There was a significant interaction between the modern food pattern and diabetes medication use in men. No association between the traditional dietary pattern and poor glycaemic control was found in either men or women. 
Table 1 Sample characteristics by gender among people with diabetes attending Qatar Biobank study ( $n$ 1000)

\begin{tabular}{|c|c|c|c|c|c|}
\hline & \multicolumn{2}{|c|}{ Male $(n 500)$} & \multicolumn{2}{|c|}{ Female $(n 500)$} & \multirow[b]{2}{*}{$P$-value } \\
\hline & $n$ & $\%$ & $n$ & $\%$ & \\
\hline Age & & & & & 0.17 \\
\hline Mean & & & & & \\
\hline SD & & & & & \\
\hline Education & & & & & $<0.001$ \\
\hline Low & 142 & 28.5 & 249 & $49 \cdot 8$ & \\
\hline Medium & 72 & 14.4 & 66 & $13 \cdot 2$ & \\
\hline High & 285 & $57 \cdot 1$ & 185 & $37 \cdot 0$ & \\
\hline Smoking & & & & & $<0.001$ \\
\hline Non & 222 & 44.4 & 482 & $96 \cdot 4$ & \\
\hline Smoker & 122 & 24.4 & 10 & $2 \cdot 0$ & \\
\hline Ex-smoker & 156 & 31.2 & 8 & 1.6 & \\
\hline Leisure time physical activity (MET h/week) & & & & & $<0.001$ \\
\hline Mean & & & & & \\
\hline SD & & & & & \\
\hline BMI $\left(\mathrm{kg} / \mathrm{m}^{2}\right)$ & & & & & $<0.001$ \\
\hline Mean & & & & & \\
\hline SD & & & & & \\
\hline BMI categories & & & & & $<0.001$ \\
\hline Normal & 64 & $12 \cdot 8$ & 23 & 4.6 & \\
\hline Overweight & 216 & $43 \cdot 2$ & 125 & $25 \cdot 0$ & \\
\hline Obese & 220 & $44 \cdot 0$ & 352 & $70 \cdot 4$ & \\
\hline Supplement use & 306 & $61 \cdot 2$ & 366 & $73 \cdot 2$ & $<0.001$ \\
\hline Vitamin D and Ca use & 138 & $27 \cdot 6$ & 249 & $49 \cdot 8$ & $<0.001$ \\
\hline Vegetable intake (times/week) & & & & & 0.44 \\
\hline Mean & & & & & \\
\hline $\mathrm{SD}$ & & & & & \\
\hline Fruit intake (times/week) & & & & & 0.35 \\
\hline Mean & & & & & \\
\hline SD & & & & & \\
\hline C-peptide (ng/ml) & & & & & 0.72 \\
\hline Mean & & & & & \\
\hline SD & & & & & \\
\hline $\mathrm{HbA} 1 \mathrm{C}(\%)$ & & & & & 0.19 \\
\hline Mean & & & & & \\
\hline SD & & & & & \\
\hline Hypertension & 197 & $39 \cdot 4$ & 219 & 43.8 & 0.16 \\
\hline $\mathrm{HbA} 1 \mathrm{C} \geq 7$ & 304 & $60 \cdot 8$ & 272 & 54.4 & 0.041 \\
\hline On diet & 371 & 74.2 & 374 & $74 \cdot 8$ & 0.83 \\
\hline Insulin use & 128 & $25 \cdot 6$ & 149 & $29 \cdot 8$ & 0.14 \\
\hline Diabetes medication other than insulin & 348 & $69 \cdot 6$ & 350 & $70 \cdot 0$ & 0.89 \\
\hline Not on any diabetes medicine & 84 & $16 \cdot 8$ & 96 & $19 \cdot 2$ & 0.32 \\
\hline Hypertension medication use & 161 & 32.2 & 184 & $36 \cdot \overline{8}$ & 0.13 \\
\hline
\end{tabular}

The three main dietary patterns identified in this study are comparable to other patterns identified in the region $^{(26-28)}$. The relationship between age and dietary pattern is also supported by other studies ${ }^{(29)}$. For example, young participants were more likely to follow the modern dietary pattern, while older participants were more likely to follow the prudent or traditional patterns. The traditional dietary pattern was characterised by high intake of biryani, chicken/meat/fish-mixed dish, fast food, Asian noodle, red meat and white rice. Those had high intake of traditional intake of traditional pattern were more likely to be smokers but less likely to use dietary supplement. The positive association between education and the modern dietary pattern is also found in other studies ${ }^{(30)}$.

The finding of an inverse association between the modern dietary pattern and poor glycaemic control in our study was inconsistent with other studies. In general, a modern dietary pattern characterised by high intake of processed energy-dense food is related to poor glycaemic control $^{(17)}$. A cross-sectional study in the UAE found that fast-food consumption more than once a week had an OR of 1.83 (95\% CI 1.02, 3.3) for poor glycaemic control among diabetic patients ${ }^{(17)}$. However, in our study, high consumption of the modern dietary pattern was positively associated with poor glycaemic control among men not taking diabetes medication. It implies that the inverse association between the modern dietary pattern and poor glycaemic control could be due to diabetes medication. In fact, among those with high intake of the modern dietary pattern, the prevalence of insulin use was 30.8\% despite a low mean age ( $46 \cdot 8$ years). It raises a significant public health message that younger diabetic men take medication to control diabetes but eat more unhealthy food. It is also possible that men who consumed the modern dietary 
Dietary pattern and glycaemic control

Table 2 Factor loadings of dietary patterns

\begin{tabular}{|c|c|c|c|}
\hline & Modern dietary pattern & Prudent pattern & Traditional pattern \\
\hline Croissant & 0.64 & -0.01 & 0.14 \\
\hline White bread & 0.56 & 0.07 & 0.26 \\
\hline Cheese & 0.55 & 0.27 & 0.07 \\
\hline Breakfast Cereal & 0.54 & 0.16 & -0.03 \\
\hline Butter & 0.51 & 0.07 & 0.10 \\
\hline Ice cream & 0.44 & -0.06 & 0.29 \\
\hline Other bread & 0.43 & 0.01 & 0.09 \\
\hline Milk & 0.39 & 0.14 & -0.07 \\
\hline Milk added to cereal & 0.39 & 0.14 & -0.11 \\
\hline Zaatar fatayer & 0.39 & 0.20 & 0.24 \\
\hline Chocolate & 0.39 & -0.02 & 0.33 \\
\hline Soft drink & 0.38 & -0.14 & 0.36 \\
\hline Tea & 0.34 & 0.28 & 0.04 \\
\hline Milk shakes & 0.31 & 0.05 & 0.10 \\
\hline Coffee & 0.28 & 0.27 & 0.07 \\
\hline Salad and raw vegetables & 0.11 & 0.68 & 0.01 \\
\hline Fresh fruit & 0.03 & 0.62 & 0.01 \\
\hline Canned/dried fruit and dates & -0.01 & 0.55 & 0.02 \\
\hline Salad and cooked vegetables & 0.29 & 0.50 & 0.22 \\
\hline Yoghurt & 0.34 & 0.47 & -0.01 \\
\hline Grilled/fried/baked Fish & -0.05 & 0.46 & 0.20 \\
\hline Fish & -0.05 & 0.46 & 0.28 \\
\hline Fresh fruit juice & 0.11 & 0.46 & 0.12 \\
\hline Soups/starters & 0.41 & 0.46 & 0.22 \\
\hline Brown bread & 0.33 & 0.36 & -0.09 \\
\hline Arabic/Iranian bread & 0.19 & 0.36 & 0.19 \\
\hline Nuts & 0.29 & 0.34 & 0.14 \\
\hline Eggs & 0.07 & 0.24 & 0.19 \\
\hline Chicken/meat/fish-mixed dish & 0.19 & 0.31 & 0.64 \\
\hline Biryani & 0.04 & 0.12 & 0.63 \\
\hline Fast food & 0.51 & -0.07 & 0.53 \\
\hline Asian noodle & 0.08 & 0.01 & 0.51 \\
\hline Red meat & 0.03 & 0.20 & 0.51 \\
\hline Lasagna & 0.13 & -0.08 & 0.50 \\
\hline White rice & -0.02 & 0.20 & 0.45 \\
\hline Chicken & 0.03 & 0.19 & 0.44 \\
\hline Desserts & 0.37 & 0.13 & 0.41 \\
\hline Potato & 0.07 & 0.12 & 0.33 \\
\hline Variance explained (\%) & $10 \cdot 8$ & $9 \cdot 3$ & 8.9 \\
\hline
\end{tabular}

Table 3 Association between dietary patterns and poor glycaemic control among people with diabetes attending Qatar Biobank study ( $n$ 1000)

\begin{tabular}{|c|c|c|c|c|c|c|c|c|c|}
\hline & \multicolumn{2}{|c|}{$\begin{array}{l}\text { Modern dietary } \\
\text { pattern }\end{array}$} & \multirow[b]{2}{*}{$P$} & \multicolumn{2}{|c|}{ Prudent pattern } & \multirow[b]{2}{*}{$P$} & \multicolumn{2}{|c|}{ Traditional pattern } & \multirow[b]{2}{*}{$P$} \\
\hline & OR & $95 \% \mathrm{Cl}$ & & OR & $95 \% \mathrm{Cl}$ & & OR & $95 \% \mathrm{Cl}$ & \\
\hline \multicolumn{10}{|l|}{ Both genders } \\
\hline Model $1^{*}$ & 0.82 & $0.72,0.94$ & 0.005 & $1 \cdot 10$ & $0.96,1.26$ & 0.159 & 1.04 & $0.91,1.19$ & 0.544 \\
\hline Model $2 \dagger$ & 0.80 & $0.68,0.93$ & 0.005 & 1.13 & $0.97,1.31$ & 0.107 & 1.01 & $0.87,1.18$ & 0.864 \\
\hline Diabetes duration (model 2) & & & $0.808 \ddagger$ & & & $0.174 \ddagger$ & & & $0.230 \ddagger$ \\
\hline$\geq 10$ years & 0.68 & $0.52,0.89$ & 0.006 & 1.08 & $0.85,1.38$ & 0.535 & $1 \cdot 19$ & $0.89,1.59$ & 0.247 \\
\hline$<10$ years & 0.83 & $0.63,1.08$ & 0.160 & 1.27 & $0.98,1.64$ & 0.066 & 0.86 & $0.68,1.11$ & 0.248 \\
\hline \multicolumn{10}{|l|}{ Men } \\
\hline Model $1^{*}$ & 0.80 & $0.65,0.99$ & 0.039 & 1.01 & $0.83,1.23$ & 0.906 & $1 \cdot 14$ & $0.94,1.39$ & $0 \cdot 171$ \\
\hline Model 2† & 0.86 & $0.68,1.08$ & 0.187 & 1.11 & $0.90,1.37$ & 0.331 & 1.02 & $0.83,1.26$ & 0.841 \\
\hline \multicolumn{10}{|l|}{ Women } \\
\hline Model $1^{*}$ & 0.84 & $0.70,1.01$ & 0.071 & $1 \cdot 16$ & $0.96,1.40$ & 0.141 & 0.95 & $0.78,1.15$ & 0.581 \\
\hline Model 2† & 0.76 & $0.61,0.95$ & 0.014 & 1.14 & $0.91,1.42$ & 0.263 & 1.00 & $0.80,1.25$ & 0.971 \\
\hline
\end{tabular}

*Model 1 adjusted for age and gender (except gender-specific models).

†Model 2 further for education, smoking, physical activity, BMI (continuous) and hypertension.

$\ddagger P$ values for interactions between diabetes duration and dietary patterns. 
(a)

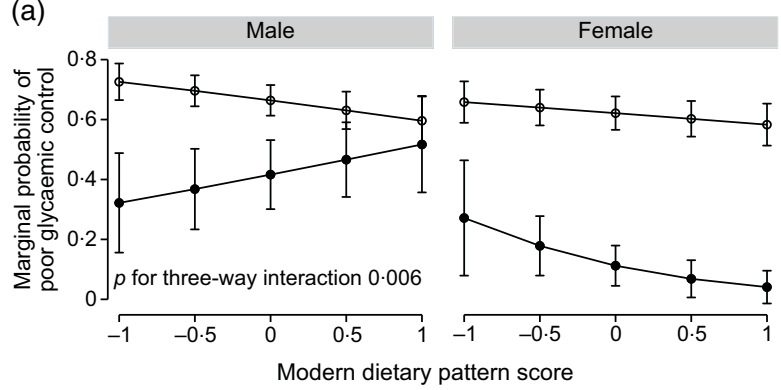

(b)

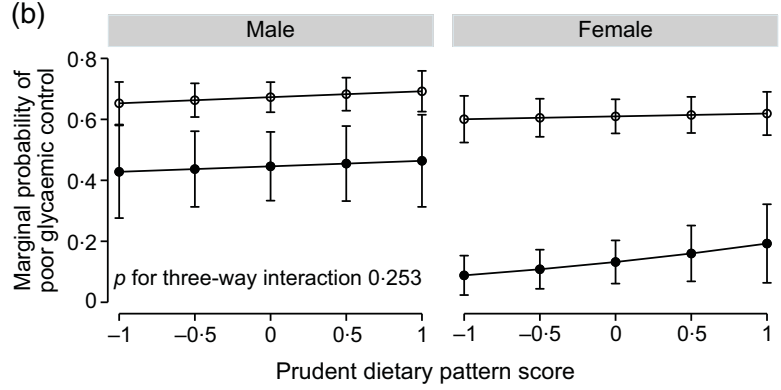

(c)

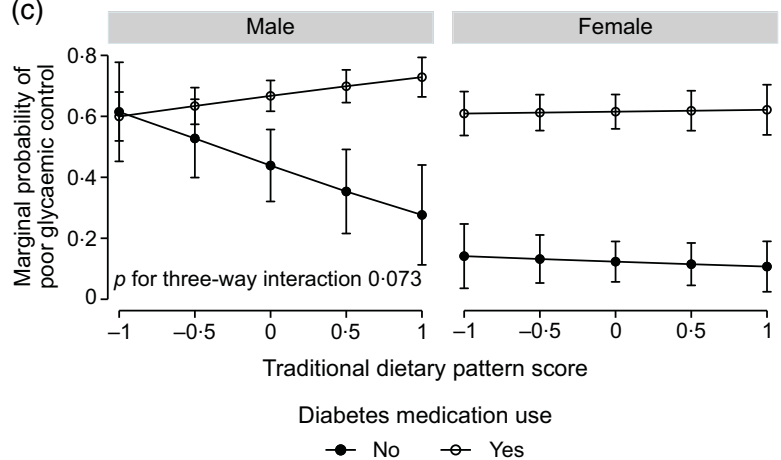

Fig. 1 Interaction between dietary patterns, diabetes medication use and gender in relation to poor glycaemic control among people with diabetes attending Qatar Biobank Study $(n 1000)$. Models adjusted for age, smoking, physical activity, education, BMI (continuous) and hypertension

pattern were taking insulin due to poor glycaemic control resulting from the unhealthier diet.

It is worth noting that women who were not taking diabetes medication had a low prevalence of poor glycaemic control (Fig. 1). However, men who were not taking diabetes medication had a high prevalence of poor glycaemic control. This suggests that women who were not taking diabetes medication might have a healthier lifestyle and diet. Despite this gender difference, women still had a high prevalence of poor glycaemic control, which is also seen in other neighbouring countries ${ }^{(31)}$.

A high prevalence of poor glycaemic control was observed in our study. This is consistent with findings from other Arabic countries including Kuwait (78.8\%) and Saudi Arabia $(67 \cdot 7 \%)^{(7)}$. The reason for the high prevalence of poor glycaemic control is unknown. It is unlikely due to the lack of diabetes medication or access to the medical service. In fact, a high proportion of the participants were on diabetes medication (only $16.8 \%$ men and $19.2 \%$ women were not on diabetes medication). A high burden of obesity in the region and sedentary behaviour could be the contributing factors. In our sample, the mean leisure time physical activity was very low (15.1 MET h/week in men and 5.8 MET h/week in women). Furthermore, unhealthy dietary habit (e.g. the mean sugar consumption in Qatar was $153 \mathrm{~g} / \mathrm{d}$ ) can also be a significant contributing factor. Sugar, rice and cooking oil are subsidised in Qatar. It is unknown to what degree this policy contributes to the burden of obesity and diabetes. Based on the factor loadings, modern dietary pattern had a high consumption of sugary food (e.g. ice cream, soft drink, dessert and chocolate). The main limitation of our study is the use of the FFQ without specifying portion sizes. We were unable to obtain data about the quantities of consumed foods and unable to adjust for energy intake in the analyses. However, the FFQ was adopted from the EPIC study with the incorporation of local food items. The limitations of FFQ have been well known (e.g. inaccurate measure of nutrients intake and limited number of food items included). On the other hand, FFQ can reflect the long-term habitual intake and can be used to rank individuals in large epidemiological studies. The FFQ used in the EPIC has been shown to have an acceptable relative validity ${ }^{(32)}$. The second limitation is the cross-sectional study design. Although we cannot make any conclusion about the causation, the findings of the current dietary patterns have strong public health significance. Third, we did not have detailed information on diabetes medication use (e.g. type and dose). However, the interaction between dietary pattern and diabetes medication use in relation to glycaemic control is unlikely changed if we had detailed information of diabetes medication use. Last, the three patterns explained only $14.5 \%$ of the variance. However, it is common that in population-based studies, dietary patterns using factor analysis cannot explain most of the variation of the whole diet. The amount of variance explained by the dietary patterns depends on the number of food groups used to construct the dietary patterns: the higher the number of food groups, the lower the variance explained by the dietary patterns. We used thirty-eight food groups in our analysis, which is higher than other studies. The thirty-eight food groups most closely represented foods commonly consumed in Qatar. The study's strength is our detailed information of disease history and lifestyle factors. Furthermore, blood samples were measured for HbA1C.

\section{Conclusion}

Poor glycaemic control is common in Qatari adults diagnosed with diabetes. Overall, the modern dietary pattern was inversely associated with poor glycaemic control, and this is likely due to the use of medication. In men who were not taking diabetes medication, the modern dietary pattern was positively associated, while the traditional 
pattern was inversely associated with poor glycaemic control. Promoting healthy eating should be encouraged especially among those taking diabetes medications.

\section{Acknowledgements}

Acknowledgements: We thank Qatar Biobank for providing us with the data needed for this research paper. We are also very grateful to all the participants of Qatar Biobank Study. Financial support: There is no financial support provided. Conflict of interest: The authors have no relevant financial interest in this article. Authorship: Conceptualisation: Z.S. Methodology: Z.S., M.A., A.O., R.K., G.A. and H.B. Formal analysis: Z.S. Resources: Z.S. Writing-original draft preparation: Z.S., M.A., A.O., R.K., G.A. and H.B. Writing, reviewing and editing: Z.S., M.A., A.O., R.K., G.A. and H.B. Supervision: Z.S. Project administration: Z.S. All authors read and approved the final version of the manuscript. Ethics of buman subject participation: This study was conducted according to the guidelines laid down in the Declaration of Helsinki. Ethical approval of QBB was obtained from the Ethics Committee of Hamad Medical Corporation in 2011 then continued with the QBB Institutional Review Board (IRB) from 2017. All participants gave written informed consent before participation. The current study was approved under the IRB exempted category (Ex-2019-RES-ACC-01630086) by the QBB.

\section{Supplementary material}

For supplementary material accompanying this paper visit https://doi.org/10.1017/S1368980020003572

\section{References}

1. International Diabetes Federation (2017) IDF Diabetes Atlas, 8th ed. Brussels. Available from: www.diabetesatlas.org (accessed July 2019).

2. Al Thani A, Fthenou E, Paparrodopoulos S et al. (2019) Qatar Biobank Cohort Study: study design and first results. Am J Epidemiol 188, 1420-1433.

3. Bener A \& Al-Hamaq AO (2016) Predictions burden of diabetes and economics cost: contributing risk factors of changing disease prevalence and its pandemic impact to Qatar. Exp Clin Endocrinol Diabetes 124, 504-511.

4. Raghavan S, Vassy JL, Ho YL et al. (2019) Diabetes mellitusrelated all-cause and cardiovascular mortality in a National Cohort of adults. J Am Heart Assoc 8, e011295.

5. Fowler MJ (2008) Microvascular and macrovascular complications of diabetes. Clin Diabetes 26, 77-82.

6. Alawadi F, Abdelgadir E, Bashier A et al. (2019) Glycemic control in patients with diabetes across primary and tertiary government health sectors in the emirate of Dubai, United Arab Emirates: a five-year pattern. Oman Med J 34, 20-25.

7. Al-Rasheedi AA (2015) Glycemic control among patients with type 2 diabetes mellitus in countries of Arabic Gulf. Int J Health Sci 9, 345-350.
8. Alromaihi D, Bastawrous M \& Bastawrous D (2019) Factors affecting glycemic control among patients with type 2 diabetes in Bahrain. Babrain Medical Bull 41, 146-149.

9. Shi Z \& Badi Abou-Samra A (2019) Association of low serum magnesium with diabetes and hypertension: findings from Qatar Biobank Study. Diabetes Res Clin Pract 158, 107903.

10. Resendiz-Abarca CA, Flores-Alfaro E, Suarez-Sanchez F et al. (2019) Altered glycemic control associated with polymorphisms in the SLC22A1 (OCT1) gene in a Mexican population with type 2 diabetes mellitus treated with metformin: a cohort study. J Clin Pharmacol 59, 1384-1390.

11. de Carvalho GB, Dias-Vasconcelos NL, Santos RKF et al. (2019) Effect of different dietary patterns on glycemic control in individuals with type 2 diabetes mellitus: a systematic review. Crit Rev Food Sci Nutr 16, 1-12.

12. Teo SYM, Kanaley JA, Guelfi KJ et al. (2018) Exercise timing in type 2 diabetes mellitus: a systematic review. Med Sci Sports Exerc 50, 2387-2397.

13. Peng K, Chen G, Liu C et al. (2018) Association between smoking and glycemic control in diabetic patients: results from the risk evaluation of cancers in Chinese diabetic individuals: a longitudinal (reaction) study. J Diabetes 10, 408-418.

14. Inada S \& Koga M (2017) Alcohol consumption reduces HbA1c and glycated albumin concentrations but not 1,5-anhydroglucitol. Ann Clin Biochem 54, 631-635.

15. Sarmento RA, Antonio JP, de Miranda IL et al. (2018) Eating patterns and health outcomes in patients with type 2 diabetes. J Endocr Soc 2, 42-52.

16. Pan B, Wu Y, Yang Q et al. (2019) The impact of major dietary patterns on glycemic control, cardiovascular risk factors, and weight loss in patients with type 2 diabetes: a network meta-analysis. J Evid Based Med 12, 29-39.

17. Sadiya A \& Mnla R (2019) Impact of food pattern on glycemic control among type 2 diabetic patients: a cross-sectional study in the United Arab Emirates. Diabetes Metab Syndr Obes 12, 1143-1150.

18. Rock CL, Flatt SW, Pakiz B et al. (2014) Weight loss, glycemic control, and cardiovascular disease risk factors in response to differential diet composition in a weight loss program in type 2 diabetes: a randomized controlled trial. Diabetes Care 37, 1573-1580.

19. Schwingshackl L, Chaimani A, Hoffmann G et al. (2018) A network meta-analysis on the comparative efficacy of different dietary approaches on glycaemic control in patients with type 2 diabetes mellitus. Eur J Epidemiol 33, $157-170$

20. Al-Thani M, Al-Thani AA, Al-Mahdi N et al. (2017) An overview of food patterns and diet quality in Qatar: findings from the National Household Income Expenditure Survey. Cureus 9, e1249.

21. Donnelly TT, Fung TS \& Al-Thani ABM (2018) Fostering active living and healthy eating through understanding physical activity and dietary behaviours of Arabic-speaking adults: a cross-sectional study from the Middle East. BMJ Open 8, e019980.

22. Ullah E, Mall R, Rawi R et al. (2018) Correction to: harnessing Qatar Biobank to understand type 2 diabetes and obesity in adult Qataris from the first Qatar Biobank Project. J Transl Med 16, 283.

23. Martínez MLR, Gómez-Díaz RA, González ALV et al. (2020) Association between glycemic control and dietary patterns in patients with type 2 diabetes in a Mexican institute. Nutrition, 110901.

24. Newby PK \& Tucker KL (2004) Empirically derived eating patterns using factor or cluster analysis: a review. Nutr Rev 62, 177-203.

25. Ibrahim AA, Qamar B, Fituri S et al. (2019) Association between soft drink consumption and asthma among Qatari adults. Nutrients 11, 606. 
26. Esmaillzadeh A, Kimiagar M, Mehrabi Y et al. (2007) Dietary patterns and markers of systemic inflammation among Iranian women. J Nutr 137, 992-998.

27. Abu-Saad K, Murad H, Lubin F et al. (2012) Jews and Arabs in the same region in Israel exhibit major differences in dietary patterns. J Nutr 142, 2175-2181.

28. Naja F, Nasreddine L, Itani L et al. (2013) Association between dietary patterns and the risk of metabolic syndrome among Lebanese adults. Eur J Nutr 52, 97-105.

29. Naidoo N, van Dam RM, Ng S et al. (2017) Determinants of eating at local and western fast-food venues in an urban Asian population: a mixed methods approach. Int J Behav Nutr Phys Act 14, 69.

30. Hidaka BH, Hester CM, Bridges KM et al. (2018) Fast food consumption is associated with higher education in women, but not men, among older adults in urban safety-net clinics: a cross-sectional survey. Prev Med Rep 12, 148-151.

31. Alzaheb RA \& Altemani AH (2018) The prevalence and determinants of poor glycemic control among adults with type 2 diabetes mellitus in Saudi Arabia. Diabetes Metab Syndr Obes 11, 15-21.

32. Kroke A, Klipstein-Grobusch K, Voss S et al. (1999) Validation of a self-administered food-frequency questionnaire administered in the European prospective investigation into Cancer and Nutrition (EPIC) Study: comparison of energy, protein, and macronutrient intakes estimated with the doubly labeled water, urinary nitrogen, and repeated 24 h dietary recall methods. Am J Clin Nutr $\mathbf{7 0}$, 439-447. 